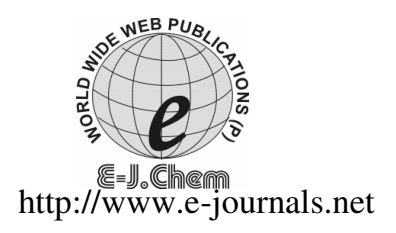

ISSN: 0973-4945; CODEN ECJHAO

E-Journal of Chemistry

2011, 8(4), 1859-1863

\title{
$L$-Proline as an Efficient Catalyst for Synthesis of Aldimines at Ambient Temperature Condition
}

\author{
S. S. KOTTAWAR, S. V. GOSWAMI, P. B. THORAT and S. R. BHUSARE* \\ Department of Chemistry \\ Dnyanopasak College, Parbhani-431401, MS, India \\ bhusare71@yahoo.com
}

Received 26 September 2010; Accepted 21 November 2010

\begin{abstract}
Some new aldimines were synthesized from substituted 2-amino thiazoles and different aromatic aldehydes using $L$-proline as an efficient catalyst. The structure elucidation of aldimines has been made on the basis of elemental analysis and spectral data. The easy work up, higher yield and shorter reaction time are the advantages of the method.
\end{abstract}

Keywords: 2-Amino thiazole, Aldehydes, $L$-Proline, Aldimines

\section{Introduction}

Utility of aldimines lies in their potent biological activity and their usefulness as a starting material for the synthesis of various heterocyclic compounds such as azetidinones and thiazolidinones. One interesting role of imines (Schiff bases) is as intermediate in the biologically important transamination reaction. In living systems the amino group of an amino acid is transferred to the carbonyl group of another molecule, promoted by an enzyme called transaminase. All the important transaminase enzymes appear to have common coenzymes, pyridoxal phosphate.

The Schiff bases constitute one of the most active class of the compounds possessing diversified biological activity such as antitubercular ${ }^{1}$, anticancer ${ }^{2}$, antibacterial ${ }^{3}$, antifungal ${ }^{3}$, analgesic ${ }^{4}$, CNS depressant ${ }^{4}$, anti-inflammatory ${ }^{5}$, anticonvulsant ${ }^{6}$, insecticidal ${ }^{7}$ and plant growth inhibitors ${ }^{8}$. The biological transformation is not simple transfer of an amino group from one molecule to another. The carbonyl group of the coenzyme pyridoxal actually forms an imine with amino group of the donor amino acid. Subsequent reaction of this adducts with the "acceptor" carbonyl compound results in the formation of a new amino acid and regeneration of the pyridoxal fragment. Pyridoxal is the carrier of the amino group from one reactant to the other where in the first step, the Schiff bases formation occurs. 
Literature survey reveals that various aldimines attracted considerable attention as they are also endowed with wide range of pharmaceutical activities. Its reactivity is greatly influenced by different substituent. Aldimines and its derivatives possess different therapeutic activities.

Proline and its derivatives are often used as catalysts in organic reactions. The CBS reduction and proline catalyzed aldol condensation are prominent examples. $L$-Proline is an osmoprotectant and therefore is used in many pharmaceutical, biotechnological applications. Recently ${ }^{9}$, the use of $L$-Proline as an organo-catalyst for the multicomponent Hantzsch reaction under benign reaction conditions is reported. $L$-Proline is a very effective, environmentally friendly catalyst for the four component condensations of dimedone, ethylacetoacetate, aromatic aldehyde and ammonium acetate to form polyhydroquinoline in excellent yields ${ }^{9}$.

Here in we report the synthesis of several new heterocyclic aldimines first time using $L$-proline as organocatalyst from various substituted 2 -amino thiazoles and aromatic aldehydes. Our results demonstrate that $L$-proline is very effective, environmentally friendly catalyst for the condensation of aromatic aldehyde and thiazole to form the aldimines in excellent yield (Scheme 1).<smiles>[R]C1=NC(/N=C/c2cc(C=O)ccc2[R])SC1</smiles>

Scheme 1. Reaction conditions: (a) Methanol, L-proline (10 mol\%), 80-85\%, 4-6 h.

\section{Experimental}

Melting points were determined in open capillary tube and are uncorrected. The purity of the compounds has been checked by TLC. The IR spectra were recorded on Varian FTIR 640 spectrometer. ${ }^{1} \mathrm{H}$ NMR and ${ }^{13} \mathrm{C}$ NMR spectra were recorded on Burker $400 \mathrm{MHz}$ spectrometer in $\mathrm{CDCl}_{3}$ as a solvent and TMS as an internal standard.

General procedure for the synthesis of aldimines (3a-j)

A mixture of equimolar quantities of substituted benzaldehydes $1(10 \mathrm{mmol})$ and substituted 2-amino thiazole $2(10 \mathrm{mmol})$ in methanol was stirred at room temperature and added catalytic amount of $L$-Proline (10 mole\%). Reaction mixture was stirred till the completion of reaction at room temperature as indicated by TLC (ethyl acetate: $n$-hexane, 2:8). After the completion of reaction, the reaction mixture was concentrated and added water. Solid was precipitated out and filtered to get crude compound. Crude compound was crystallized from ethanol to get pure compound (3a-j) (Table 1$)$.

Table 1. Synthesis of aldimines (3a-j)

\begin{tabular}{cllccc}
\hline Entry & \multicolumn{1}{c}{$\mathrm{R}$} & \multicolumn{1}{c}{$\mathrm{R}_{1}$} & $\mathbf{3 a - j}$ & Time, $\mathrm{h}$ & Yield, \% \\
\hline 1. & 3,4,5-Trimethoxy & 4-Phenyl & $\mathbf{3 a}$ & 4.5 & 85 \\
2. & 3,4,5-Trimethoxy & 4-Chlorophenyl & $\mathbf{3 b}$ & 4.5 & 82 \\
3. & 5-Bromo-2-hydroxy & 4-Naphthalene & $\mathbf{3 c}$ & 5.5 & 76 \\
4. & 5-Bromo-2-hydroxy & 3-Nitrophenyl & $\mathbf{3 d}$ & 5.0 & 78 \\
5. & 5-Bromo-2-hydroxy & 4-Chlorophenyl & $\mathbf{3 e}$ & 5.0 & 75 \\
6. & 5-Bromo-2-hydroxy & 4-Phenyl & $\mathbf{3 f}$ & 5.0 & 79 \\
7. & 3-Chloro & 4-Naphthalene & $\mathbf{3 g}$ & 4.0 & 84 \\
8. & 3-Chloro & 4-Chlorophenyl & $\mathbf{3 h}$ & 5.5 & 82 \\
9. & 2-Hydroxy & 3-Nitrophenyl & $\mathbf{3 i}$ & 6.0 & 85 \\
10. & 2-Hydroxy & 4-Chlorophenyl & $\mathbf{3 j}$ & 4.5 & 83 \\
\hline
\end{tabular}




\section{4-Phenyl-N-(3,4,5-trimetoxybenzylidene)thiazol-2-amine (3a)}

${ }^{1} \mathrm{H}$ NMR: $\delta 3.61\left(\mathrm{~s}, 9 \mathrm{H}, \mathrm{CH}_{3}\right), 7.1-7.8(\mathrm{~m}, 7 \mathrm{H}, \mathrm{ArH}), 8.41(\mathrm{~s}, 1 \mathrm{H}$, thiazole $\mathrm{H}), 9.35(\mathrm{~s}, 1 \mathrm{H}$, $=\mathrm{CH}) ;{ }^{13} \mathrm{C}$ NMR: $\delta$ 55.4, 59.5, 103.4, 110.8, 126.4, 127.1, 128.5, 131.6, 131.8, 140.8, 150.9, 151.1, 161.2, 170.8; ESMS: 353.1 (M-1); Elemental Analysis: $\mathrm{C}_{19} \mathrm{H}_{18} \mathrm{~N}_{2} \mathrm{O}_{3} \mathrm{~S}$; Calcd. C, 64.39; H, 5.12; N, 7.90; O, 13.54; S, 9.05; Found C, 64.40; H, 5.11; N, 7.89; O, 13.56; S, 9.04; m. p. $165-167{ }^{\circ} \mathrm{C}$;

\section{4-(4-Chlorophenyl)-N-(3,4,5-trimethoxybenzylidene)thiazol-2-amine (3b)}

${ }^{1} \mathrm{H}$ NMR: $\delta$ 3.61(s, 9H, $\mathrm{CH}_{3}$ ), 7.1 (s, 2H, ArH), 7.6 (d, 2H, ArH), 7.85 (d, 2H, ArH), 8.40 (s, $1 \mathrm{H}$, thiazole $\mathrm{H}), 9.31(\mathrm{~s}, 1 \mathrm{H},=\mathrm{CH}) ;{ }^{13} \mathrm{C}$ NMR: $\delta 55.4,59.5,103.4,110.8,126.7,127.6$, 128.6, 131.6, 131.8, 140.8, 150.9, 151.1, 161.2, 170.8; ESMS: 387.1 (M-1); Elemental Analysis: $\mathrm{C}_{19} \mathrm{H}_{17} \mathrm{ClN}_{2} \mathrm{O}_{3} \mathrm{~S}$; Calcd. C, 58.68; H, 4.41; Cl, 9.12; N, 7.20; O, 12.35; S, 8.25; Found C, 58.69; H, 4.42; Cl, 9.13; N, 7.21; O, 12.36; S, 8.24; m. p. 179-181 ${ }^{\circ} \mathrm{C}$.

\section{4-Naphthalene-N-(2-hydroxy-5-bromobenzylidene)thiazol-2-amine (3c)}

${ }^{1} \mathrm{H}$ NMR: $\delta 6.95(\mathrm{~d}, 1 \mathrm{H}, \mathrm{ArH}), 7.4-8.0(\mathrm{~m}, 9 \mathrm{H}, \mathrm{ArH}), 8.45$ (s, 1H, thiazole H), $9.35(\mathrm{~s}, 1 \mathrm{H}$, $=\mathrm{CH}), 12.35(\mathrm{~s}, 1 \mathrm{H}, \mathrm{OH}) ;{ }^{13} \mathrm{C}$ NMR: $\delta 110.80,114.62,119.27,121.84,123.97,124.77$, $126.35,127.61,128.26,128.36,131.31,131.91,132.73,133.13,136.98,152.55,159.19$, 161.11, 170.63; ESMS: 407.5 (M-1); Elemental Analysis: $\mathrm{C}_{20} \mathrm{H}_{13} \mathrm{BrN}_{2} \mathrm{OS}$; Calcd. C, 58.69; H, 3.20; Br, 19.52; N, 6.84; O, 3.91; S, 7.83. Found C, 58.70; H, 3.19; Br, 19.50; N, 6.82; O, 3.93; S, 7.82; m. p. $169-171^{\circ} \mathrm{C}$.

\section{4-(3-Nitrophenyl)-N-(2-hydroxy-5-bromobenzylidene)thiazol-2-amine (3d)}

${ }^{1} \mathrm{H}$ NMR: 6.9 (d, 1H, ArH), 7.3 (d, 1H, ArH), 7.7- 8.3 (m, 5H, ArH), 8.7 (s, 1H, ArH), 9.41 $(\mathrm{s}, 1 \mathrm{H},=\mathrm{CH}) ;{ }^{13} \mathrm{C}$ NMR: $\delta 110.6,110.8,118.3,119.6,128.5,128.9,131.1,132.1,133.9$, 134.1, 152.4, 160.2, 161.4, 170.6; ESMS: 401.9 (M-1); Elemental Analysis: $\mathrm{C}_{16} \mathrm{H}_{10} \mathrm{BrN}_{3} \mathrm{O}_{3} \mathrm{~S}$ : Calcd. C, 47.54; H, 2.49; Br, 19.77; N, 10.39; O, 11.87; S, 7.93. Found C, 47.55; H, 2.47; Br, 19.78; N, 10.36; O, 11.89; S, 7.94; m. p. 238-240 ${ }^{\circ} \mathrm{C}$.

\section{4-(4-Chlorophenyl)-N-(2-hydroxy-5-bromobenzylidene)thiazol-2-amine (3e)}

${ }^{1} \mathrm{H}$ NMR: $\delta$ 6.95-7.91 (m, 8H, ArH), H), $9.35(\mathrm{~s}, 1 \mathrm{H},=\mathrm{CH}), 12.35(\mathrm{~s}, 1 \mathrm{H}, \mathrm{OH}) ;{ }^{13} \mathrm{C}$ NMR: $\delta$ 110.6, 110.8, 118.3, 119.6, 121.5, 122.1, 128.4, 131.1, 132.3, 132.9, 134.3, 150.1, 152.5, 160.1, 161.4, 170.6; ESMS: 390.9 (M-1); Elemental Analysis: $\mathrm{C}_{16} \mathrm{H}_{10} \mathrm{ClN}_{2} \mathrm{OS}$ : Calcd. C, 48.81; H, 2.56; Br, 20.30; Cl, 9.01; N, 7.12; O, 4.06; S, 8.14; Found C, 48.82; H, 2.58; Br, 20.31; Cl, 9.03; N, 7.11; O, 4.07; S, 8.12; m. p.198-201 ${ }^{\circ} \mathrm{C}$.

\section{4-Phenyl-N-(2-hydroxy-5-bromobenzylidene)thiazol-2-amine (3f)}

${ }^{1} \mathrm{H}$ NMR: $\delta 6.91(\mathrm{~d}, 1 \mathrm{H}, \mathrm{ArH}), 7.4-7.8(\mathrm{~m}, 7 \mathrm{H}, \mathrm{ArH}), 8.44(\mathrm{~s}, 1 \mathrm{H}$, thiazole $\mathrm{H}), 9.32(\mathrm{~s}, 1 \mathrm{H}$, $=\mathrm{CH}) ;{ }^{13} \mathrm{C}$ NMR: $\delta$ 110.5, 110.8, 118.4 119.7, 126.8, 127.4, 128.6, 130.4, 131.9, 134.2, 160.2, 161.4, 170.6; ESMS: 356.9 (M-1); Elemental Analysis: $\mathrm{C}_{16} \mathrm{H}_{11} \mathrm{BrN}_{2} \mathrm{OS}$ : Calcd. C, 53.49; H, 3.09; Br, 22.24; N, 7.80; O, 4.45; S, 8.93; Found C, 53.48; H, 3.11; Br, 22.23; N, $7.81 ; \mathrm{O}, 4.44 ; \mathrm{S}, 8.94 ;$ m. p. $130-131^{\circ} \mathrm{C}$.

\section{4-Naphthalene-N-(3-chlorobenzylidene)thiazol-2-amine (3g)}

${ }^{1} \mathrm{H}$ NMR: $\delta$ 7.4-8.0 (m, 11H, ArH), $8.41(\mathrm{~s}, 1 \mathrm{H}$, thiazole $\mathrm{H}), 9.35(\mathrm{~s}, 1 \mathrm{H},=\mathrm{CH}) ;{ }^{13} \mathrm{C}$ NMR: $\delta$ $110.81,114.92,119.35,121.89,123.77,124.77,126.35,126.58,128.26,131.41,131.81$, 132.93, 133.23, 136.98, 152.15, 159.09, 161.23, 170.68; ESMS: 347.1 (M-1); Elemental Analysis: $\mathrm{C}_{20} \mathrm{H}_{13} \mathrm{ClN}_{2} \mathrm{~S}$ : Calcd. C, 68.86; H, 3.76; Cl, 10.16; N, 8.03; S, 9.19; Found $\mathrm{C}$, 68.87; H, 4.78; Cl, 10.12; N, 7.02; S, 9.18; m. p.125-128 ${ }^{\circ} \mathrm{C}$. 


\section{4-(4-Chlorophenyl)-N-(3-chlorobenzylidene)thiazol-2-amine (3h)}

${ }^{1} \mathrm{H}$ NMR: $\delta$ 7.4-8.0 $(\mathrm{m}, 8 \mathrm{H}, \mathrm{ArH}), 8.40(\mathrm{~s}, 1 \mathrm{H}$, thiazole $\mathrm{H}), 9.30(\mathrm{~s}, 1 \mathrm{H},=\mathrm{CH}) ;{ }^{13} \mathrm{C}$ NMR: $\delta$ 110.8, 125.8, 125.9, 127.5, 127.6, 129.4, 130.8, 132.8, 132.9, 135.4, 159.09, 161.23, 170.68. ESMS: 331.1 (M-1); Elemental Analysis: $\mathrm{C}_{16} \mathrm{H}_{10} \mathrm{Cl}_{2} \mathrm{~N}_{2} \mathrm{~S}$ : Calcd. C, 57.67; H, 3.02; Cl, 21.28; $\mathrm{N}, 8.41 ; \mathrm{S}, 9.62$; Found C, 57.68; H, 3.03; Cl, 21.30; N, 8.38; S, 9.60; m. p. 145-147 ${ }^{\circ} \mathrm{C}$.

\section{4-(3-Nitrophenyl)-N-(2-hydroxybenzylidene)thiazol-2-amine (3i)}

${ }^{1} \mathrm{H}$ NMR: $\delta$ 7.0-8.2 (m, 7H ArH), $8.40(\mathrm{~s}, 1 \mathrm{H}$, thiazole $\mathrm{H}), 8.55(\mathrm{~s}, 1 \mathrm{H}, \mathrm{ArH}), 9.30(\mathrm{~s}, 1 \mathrm{H}$, $=\mathrm{CH}) ;{ }^{13} \mathrm{C}$ NMR: $\delta 110.8,117.5,119.4,120.7,122.5,123.2,131.5,132.2,132.6,132.9$, 133.0, 149.6, 152.5, 159.09, 161.23, 170.68; ESMS: 324.1(M-1); Elemental Analysis: $\mathrm{C}_{16} \mathrm{H}_{11} \mathrm{ClN}_{3} \mathrm{O}_{3} \mathrm{~S}$ : Calcd. C, 59.07; H, 3.41; N, 12.92; O, 14.75; S, 9.86; Found C, 58.05; H, 3.42 ; N, 12.91; O, 14.76; S, 9.85; m. p.218-220 ${ }^{\circ} \mathrm{C}$.

\section{4-(4-Chlorophenyl)-N-(2-hydroxybenzylidene)thiazol-2-amine (3j)}

${ }^{1} \mathrm{H}$ NMR: $\delta$ 6.91-7.1(m, 2H, ArH), 7.4-7.6 (m, 4H, ArH and s, 1H, thiazole H), $7.87(\mathrm{~d}, 2 \mathrm{H}$, $\mathrm{ArH}), 9.35(\mathrm{~s}, 1 \mathrm{H},=\mathrm{CH}) ;{ }^{13} \mathrm{C}$ NMR: $\delta 110.8,116.5,119.6,120.5,127.6,127.9,131.1,131.9$, 135.6, 152.5, 159.09, 161.23, 170.68; ESMS: 313.1 (M-1); Elemental Analysis: $\mathrm{C}_{16} \mathrm{H}_{11} \mathrm{ClN}_{2} \mathrm{OS}$ : Calcd. C, 61.05; H, 3.52; Cl, 11.26; N, 8.90; O, 5.08; S, 10.19; Found C, 61.06; H, 3.54; Cl, 11.27; N, 8.91; O, 5.06; S, 10.20; m. p.159-161 ${ }^{\circ} \mathrm{C}$.

\section{Results and Discussion}

The experimental procedure is very simple and easy to carry out. A mixture of aromatic aldehyde and substituted 2-amino thiazole was vigorously stirred in methanol in the presence of catalyst $L$-proline $(10 \mathrm{~mol} \%)$ at room temperature until the completion of reaction. Progress of reaction was monitored by Thin-Layer Chromatography (TLC). On completion of the reaction, concentrated the crude reaction mixture and extracted the product in ethyl acetate followed by evaporation of solvent gave crude product, which was further crystallized with ethanol to get pure product in excellent yields. All the products were characterized by melting point, ${ }^{1} \mathrm{H}$ NMR, ${ }^{13} \mathrm{C}$ NMR spectral and elemental analysis. To the best of our knowledge, this is the first approach for the synthesis of aldimines carried out in methanol in presence of $L$-Proline catalyst at ambient temperature. It can be observed that almost all reactions were complete in $4-6 \mathrm{~h}$ in methanol and $L$-Proline as a catalyst at ambient temperature. The amount of solvent used in the reaction did not have significant influence on the overall rate of the reaction and yields of products. The reaction went to completion in identical time and with the same isolated yields as for the diluted reaction mixture. This observation assumes great significance for optimizing reactor volumes during scale-up operations. A highly efficient stirring is required for the success of this reaction. The good results obtained following our simple procedure are a pleasant surprise in view of the numerous catalysts employed in organic solvents to synthesize this class of compounds.

\section{Conclusion}

In conclusion, we describe a mild and efficient method for the synthesis of aldimines by using $L$-proline as a Lewis acid catalyst method. This method not only provides an excellent complement to aldimines synthesis but also avoids the use of hazardous acids or bases and harsh reaction conditions. The advantages of this method include the use of inexpensive reagents and catalyst under mild condition and experimental operational ease.

\section{Acknowledgment}

We acknowledge Dr. P. L. More and Dr. W. N. Jadhav, Dnyanopasak College, Parbhani for providing necessary facilities and UGC, New Delhi for financial support (MRP-47-125/06). 


\section{References}

1. Marchant J R and Chothia D S, J Med Chem., 1970, 13, 335-338.

2. $\quad$ Singare M S and Ingle D B, J Indian Chem Soc., 1976, 53, 1036-1037.

3. (a) Bhusare S R, Shinde A B, Pawar R P and Vibhute Y B, Indian J Pharm Sci., 2004, 2, 228-231; (b) Bhusare S R, Ardhapure S S, Jadhav W N, Pawar R P and Vibhute Y B, Asian J Chem., 2002, 14 (1), 209-212.

4. (a) Dobaria A V, Patil J R, Padaliya J V and Parekh H H, Indian J Heterocycl Chem., 2001, 11, 115-118; (b) Nair S M and Bhattacharya I R A, Asian J Chem., 2009, 21, 504-510; (c) Shah S, Vyas R and Mehta R H, J Indian Chem Soc., 1992, 69, 590-592; (d) Parekh J, Inamdha P, Nair R, Balusa S and Chanda S, J Serb Chem Soc., 2005, 70, 1155-1161; (e) Satyanarayana V S V, Sreevani P, Sivakumar A and Vijakumar V, Arkivoc., 2008, 17, 221-223; (f) Sutariya B, Raziya S K, Mohan S and Sambasiva Rao S V, Indian J Chem., 2007, 46B, 884-887; (g) Bairagi S, Bhosale A and Deodhar M N, E-J Chem., 2009, 6(3), 759-762; (h) Mishra A P and Soni M, MetalBased Drugs, 2008, 2, 875410, 1-7.

5. Gupta J K, Biplab De and Saravanan V S, Indian J Chem., 2006, 45B, 2580-2582.

6. $\quad$ Bawa S and Suresh Kumar, Indian J Chem., 2009, 48B, 142-145.

7. Verma M, Pandeya S N, Singh K N and Stables J P, Acta Pharm., 2004, 54(1), 49-56.

8. Kozlov N S, Korotyshova G P, Rozhkora N G and Andreeva E I, Vesti Akad Navuk USSRser khim Navuk., 1986, 2, Chem Abstr.. 1987, 106, 155955.

9. Vibhute A Y, Junne S B, Gurav V M and Vibhute Y B, J Chem Pharm Res., 2010, 2(2), 300-303. 


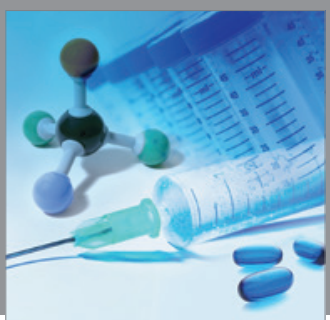

International Journal of

Medicinal Chemistry

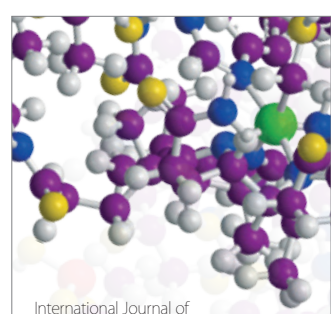

Carbohydrate Chemistry



The Scientific World Journal
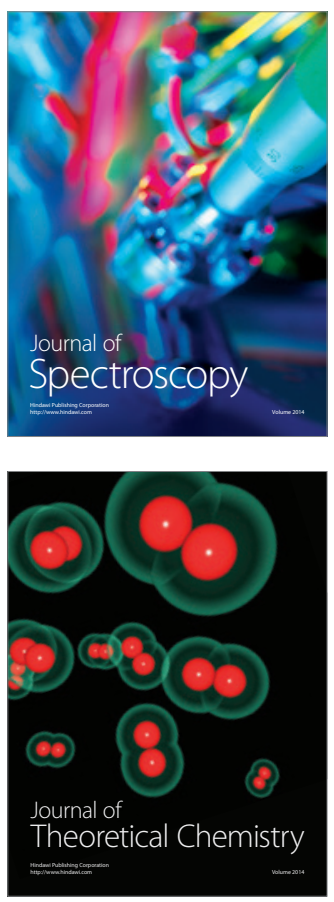
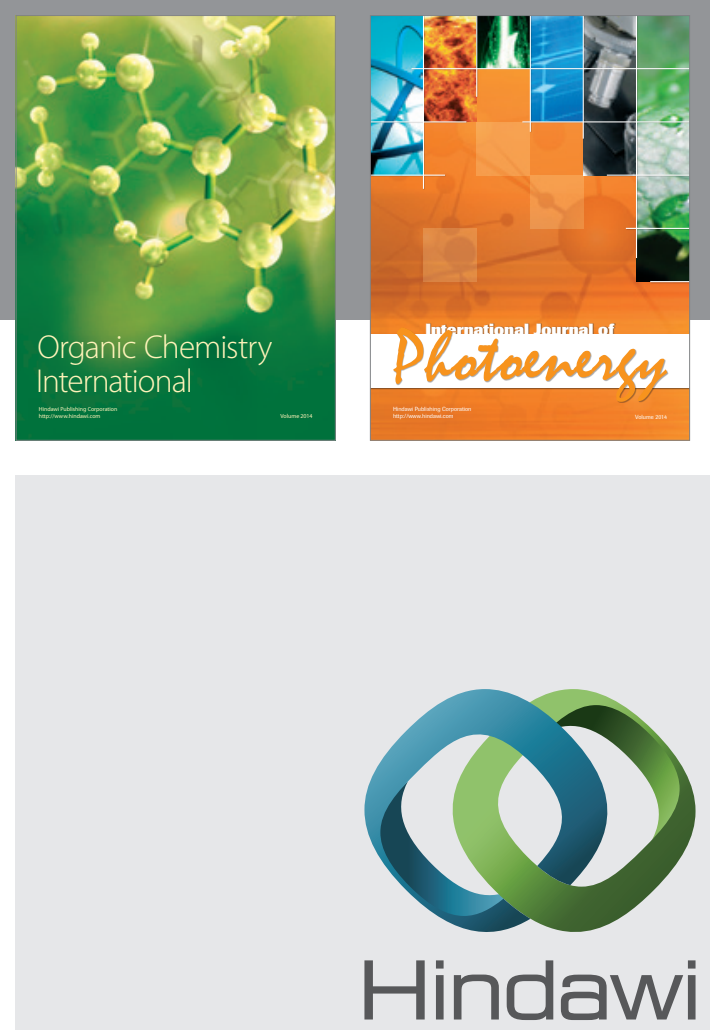

Submit your manuscripts at

http://www.hindawi.com
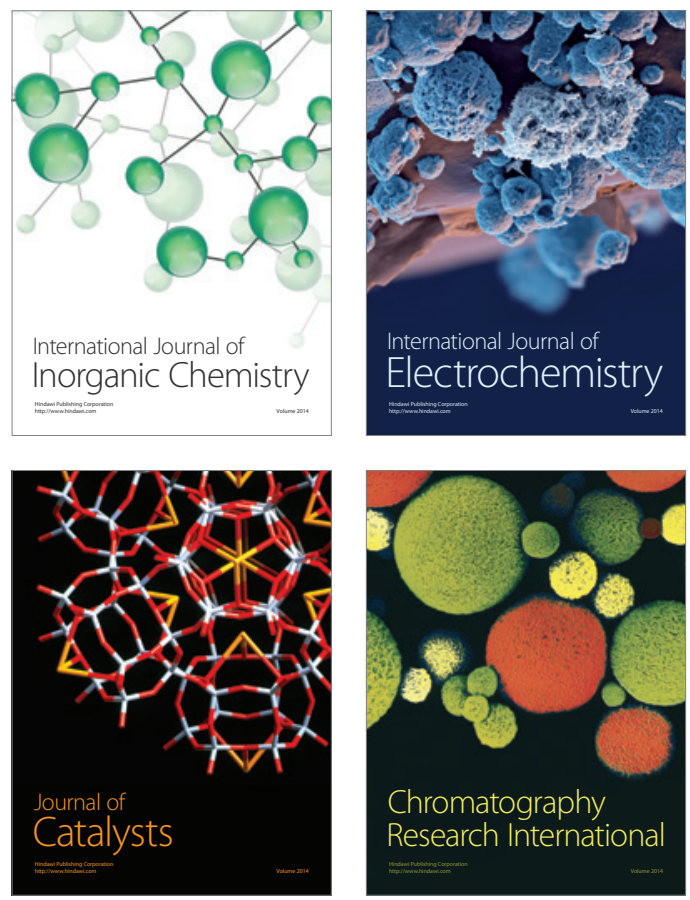
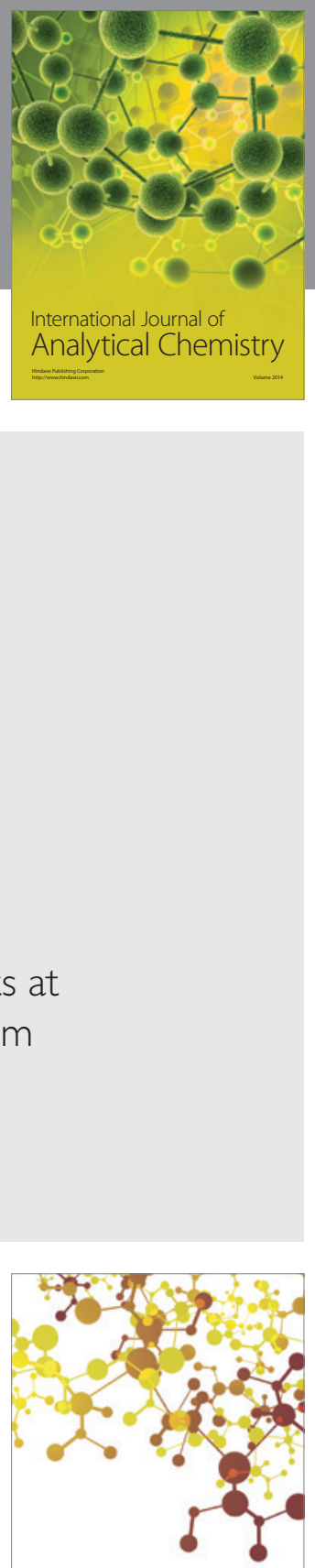

Journal of

Applied Chemistry
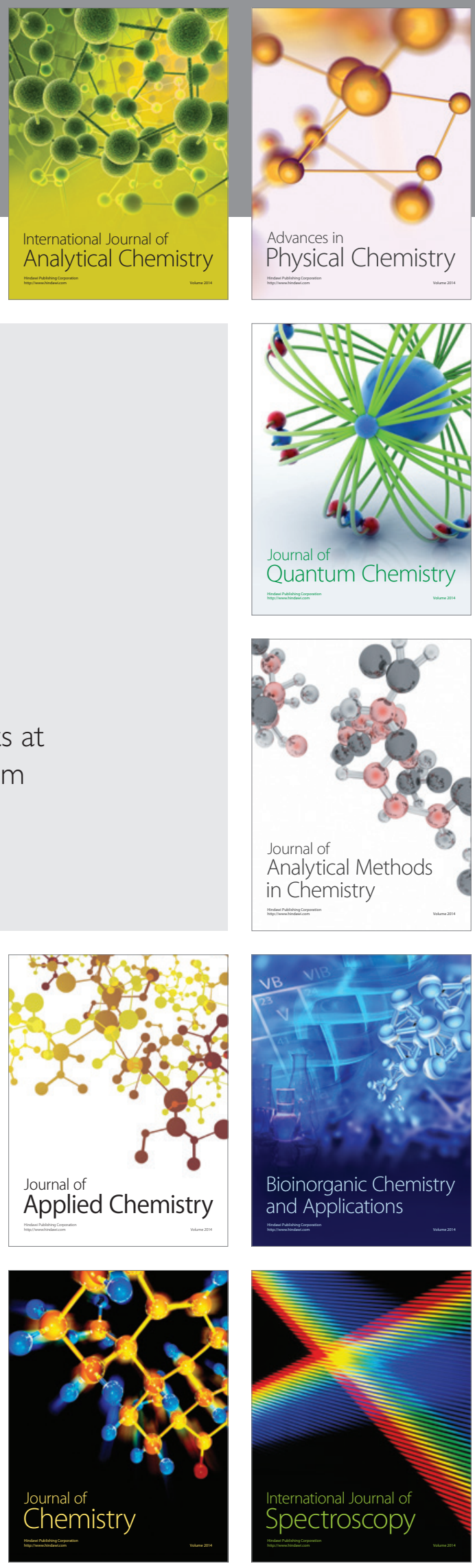